\title{
Segway Robot Designing And Simulating, Using BELBIC
}

\author{
Nazanin Moarefi ${ }^{1}$, Ali Yarahmadi ${ }^{2}$, Parisa Esfehani ${ }^{3}$, Maryam Mohabbati $^{4}$ \\ ${ }^{1,3,4}$ Technical department of Parsian higher education private institute, Qazvin \\ ${ }^{2}$ Technical department of Azad University Branch of Tehran, North
}

\begin{abstract}
Segway or human transporter is a personal transporter device that was introduced in 2002 by Dean Kamen. This device caused a good revolution in society transportation. Using of these robots is general in many countries around the world. Stability is a vital issue in Segway robots, because they could not keep their balance without a suitable controller and cannot stay balanced. In Segway system, many parameters are supervised and controlled. Various methods have been presented for their balance keeping. In this article, the BELBIC has been used for the robot moving and balancing. The results of that controller using have been stated after that.
\end{abstract}

Keywords: Segway Robot, Segway Robot Controller, Segway robot balance.

\section{Introduction}

Robotic designing uses two sensors. An accelerometer, an Electro-mechanic device that measures the Inertial Acceleration Force, while Gyroscope measures the body Angular Velocity (Sadeghian \& Masoule, 2016). Using an improved technology based on public transportation as a basic solution is one of the viewpoints. Among the various methods, Mptds can help, improving the transportaion without the car and for short trips (Gong, Wu, \& Ma, 2015; Sadeghian \& Masoule, 2016). Electronic Scooters and segways are two operator-friendly that simplify the journeys and provid suitable transportation within the cities. The aim here is the designing and making of the Segway personal transporter. It based on inverted pendulum fundament that generates a zero angle towards the vertical line during all times. It is an intelligent transporter. It uses the gyroscopic sensors to identify motion of rider (Gong et al., 2015). Segway consists of four part conatin wheel and engine, sensor, circuit platform and performance control system (Ebrahimi \& Mardany, 2015; Kim, Yoo, Bae, Kim, \& Choi, 2015). It moves forward if bent forward and vice versa. It twists right or left by the minimum deviance of handle.The technology and process that introduces this kind of movement is called dynamic stability (Wang, Lu, \& Hou, 2015). Figur 1 shows a view of segway robot. Figure 2 also shows the angles and particles f segway robot.

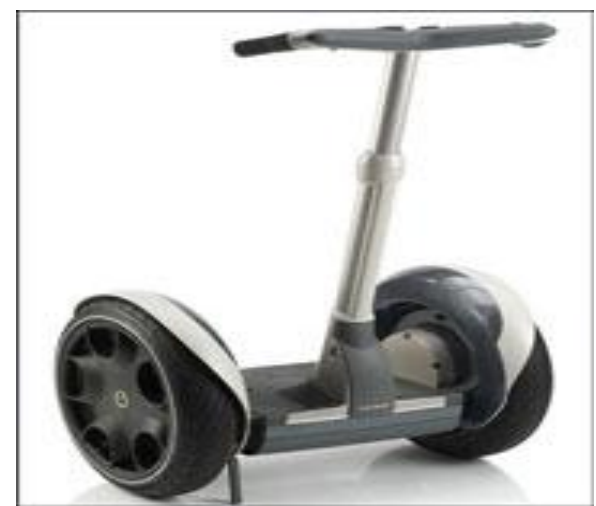

Fig1. A view of segway robot

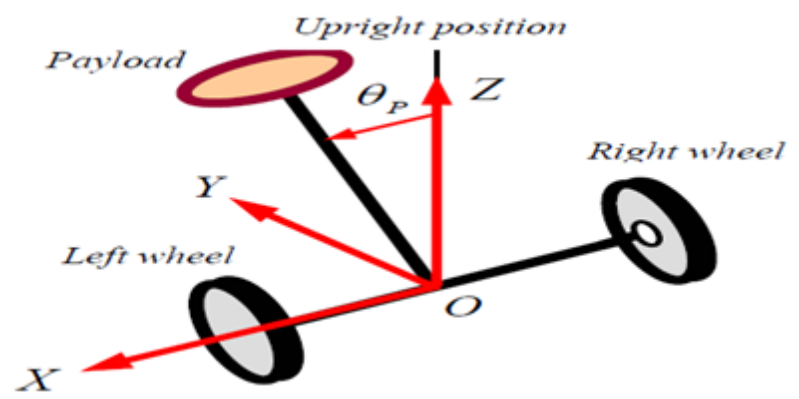

Fig2. angles and particles f segway robot 
Describing the robot states, there are mathematic relations (Ebrahimi \& Mardany, 2015). In segway system, two parameters should be controlled that are balance and rotation deflection. The balance means prevention of system falling during the forward and backward movement and rotation deflection is the regulation of steering angle during the right and left deflection (Guan, Liang, \& Gao, 2015).

\section{The Governing Equations of Segway robot}

Assessing the needed equations, figure 3 shows the robot structure.

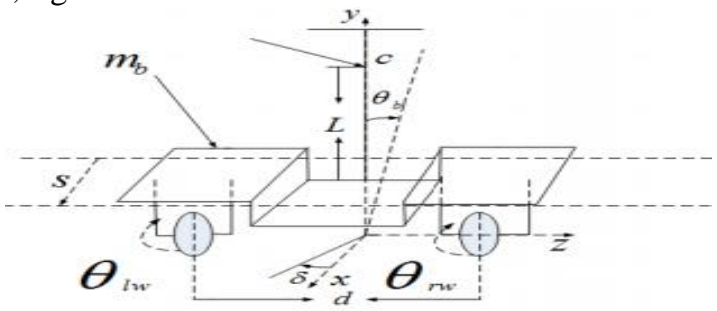

(a)

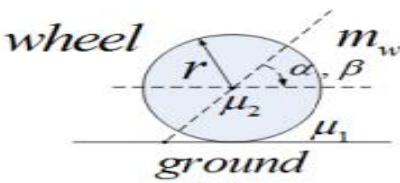

(b)

Fig3. System structure and wheels

$\mathrm{C}$ shows the robot mass center. In addition, $\mathrm{m}_{\mathrm{b}}$ is the Segway mass, $\mathrm{m}_{\mathrm{w}}$ shows the wheel mass and $\mathrm{r}$ is the wheel radial. $\mathrm{L}$ is the robot mass center and wheel axis distance. $\mathrm{N}$ is the gear in engine ratio, $\mathrm{d}$ shows the two wheels distance, $\theta_{\mathrm{b}}$ determines the stability angle, $\theta_{\mathrm{lw}}$ and $\theta_{\mathrm{rw}}$ are the movement angle of left and right wheels. Inertia momentum of body at center angle around the $Z$-axis is $\mathbf{J}_{\mathrm{vwc}}$ and $\mathrm{J}_{\mathrm{vy}}$ is the rotational inertia momentum around the $\mathrm{Y}$-axis. $\mathrm{J}_{\mathrm{y}}$ also is the rotational inertia momentum of wheel around the $\mathrm{Y}$-axis. $\mathrm{J}_{\mathrm{z}}, \mathrm{J}_{\mathrm{m}}, \mu_{1}, \mu_{2}$ and $\mathrm{s}$, are the rotational inertia momentum of wheel around the $\mathrm{z}$-axis, inertia momentum of engine rotors, friction coefficient between the earth and wheel, friction coefficient between the wheel and its axis and Deviation angle of $\delta$ with bottom part of robot respectively. The base angle of left wheel with the earth and right one are $=\theta_{\mathrm{lw}^{-}}$ $\theta_{\mathrm{b}} \alpha$ and $=\theta_{\mathrm{rw}}-\theta_{\mathrm{b}} \beta$ respectively (Wardoyo, Hendi, Sebayang, Hidayat, \& Adriansyah, 2015).

\section{The Governing Equations of Robot in Constant State}

The robot movement is explained via three section. The $\mathrm{V}_{1}$ considered velocity that is shown by operator. The $\mathrm{V}_{2}$ that is the rotation velocity in $\mathrm{Y}$-axis and the real velocity that is shown by the $\mathrm{V}_{3}$. Each section can be assessed in separate part along the $\mathrm{X}, \mathrm{Y}$ and $\mathrm{Z}$ axes.

$$
\begin{aligned}
& V_{1 x}=L \dot{\theta}_{b} \cos \theta_{b} \cos \delta \\
& V_{1 y}=-L \dot{\theta}_{b} \sin \theta_{b} \\
& V_{1 z}=L \dot{\theta}_{b} \cos \theta_{b} \sin \delta \\
& V_{2 x}=\frac{r\left(\dot{\theta}_{l w}+\dot{\theta}_{r w}\right)}{2} \cos \delta \\
& V_{2 y}=0 \\
& V_{2 z}=\sin \delta \frac{r\left(\dot{\theta}_{l w}+\dot{\theta}_{l w}\right)}{2} \\
& V_{3 x}=\frac{r\left(\dot{\theta}_{l w}-\dot{\theta}_{r w}\right)}{d} L \sin \theta_{b} \sin \delta \\
& V_{3 y}=0 \\
& V_{3 z}=L \sin \theta_{b} \cos \delta \frac{r\left(\dot{\theta}_{l w}-\dot{\theta}_{r w}\right)}{2}
\end{aligned}
$$


Calculating the total Kinetic energy, the system velocity should be explained as the summation of velocity along the different directions $\left(\mathrm{V}_{\mathrm{x}}, \mathrm{V}_{\mathrm{y}}\right.$ and $\left.\mathrm{V}_{\mathrm{z}}\right)$.

$$
\begin{aligned}
& V_{x}=V_{1 x}+V_{2 x}+V_{3 x}=L \dot{\theta}_{b} \cos \theta_{b} \cos \delta \\
& +\frac{r\left(\dot{\theta}_{l w}+\dot{\theta}_{r w}\right)}{2}+\frac{r\left(\dot{\theta}_{l w}-\dot{\theta}_{r w}\right)}{2} L \sin \theta_{b} \sin \delta \\
& V_{y}=-L \dot{\theta}_{b} \sin \theta_{b} \\
& V_{z}=V_{1 z}+V_{2 z}+V_{3 z}=L \dot{\theta}_{b} \cos \theta_{b} \sin \delta \\
& +\sin \delta \frac{r\left(\dot{\theta}_{l w}+\dot{\theta}_{r w}\right)}{2}+L \sin \theta_{b} \cos \delta \frac{r\left(\dot{\theta}_{l w}-\dot{\theta}_{r w}\right)}{2}
\end{aligned}
$$

\section{The governing equations of robot in transient state}

- Total Kinetic energy of system

Robot body consists of two part (upper and bottom). Because the engines link to the robot body, therefore the main mass of body is equal to engines mass summation plus other parts. The kinetic energy relation of wheels can be written as below:

$$
\begin{aligned}
& k_{w}=\left(k_{l w}+k_{L \mid r}+k_{L \mid z}\right)+\left(k_{r w}+k_{r \mid r}+k_{r \mid z}\right)= \\
& \frac{1}{2}\left(m_{w} r^{2}+J_{y}\right)\left(\dot{\theta}_{l w}^{2}+\dot{\theta}_{r w}^{2}\right)+\frac{1}{4} m_{w} r^{2}\left(\dot{\theta}_{l w}-\dot{\theta}_{r w}\right)^{2}
\end{aligned}
$$

Where $\mathrm{K}_{\mathrm{lw}}$ andK $\mathrm{K}_{\mathrm{rw}}$ are the Kinetic energy of left and right wheels respectively. In addition, $\mathrm{K}_{\mathrm{l|}}$ andK $\mathrm{K}_{\mathrm{r} \mid \mathrm{r}}$ are therotational Kinetic energy of each wheel. $\mathrm{K}_{\mathrm{lz}}$ andK $\mathrm{K}_{\mathrm{r} \mid \mathrm{z}}$ are the Kinetic energy of left and right wheel around the Z-axis respectively.

$$
\begin{aligned}
& k_{v}=k_{v m}+k_{v y}+k_{v w}=\frac{1}{8} m_{w} r^{2}\left(\dot{\theta}_{l w}+\dot{\theta}_{r w}\right)^{2} \\
& +\frac{1}{2}\left(m_{v} L^{2}+J_{v w c}\right) \dot{\theta}_{b}^{2}+\frac{1}{2} m_{v} L r \dot{\theta}_{b}\left(\dot{\theta}_{l w}+\dot{\theta}_{r w}\right) \cos \theta_{b} \\
& +\frac{1}{2} \frac{R^{2}}{D^{2}}\left(m_{v} L^{2} \sin ^{2} \theta_{b}+J_{v y}\right)\left(\dot{\theta}_{l w}-\dot{\theta}_{r w}\right)^{2}
\end{aligned}
$$

Where the body Kinetic energy along the center is $\mathrm{K}_{\mathrm{vw}}$. In addition, $\mathrm{K}_{\mathrm{vy}}$ is the body Kinetic energy around the $\mathrm{Y}$-axis at rotation time and $\mathrm{K}_{\mathrm{vm}}$ is the robot bottom Kinetic energy during the rotation around the $\mathrm{Y}$ axis. The Kinetic energy of engine rotors is computable via relation 15.

$$
k_{m}=\frac{1}{2} J_{m} n^{2}\left[\left(\dot{\theta}_{l w}-\dot{\theta}_{b}\right)^{2}+\left(\dot{\theta}_{r w}-\dot{\theta}_{b}\right)^{2}\right]
$$

The total Kinetic energy of system derived from three-part energy compounding $\left(\mathrm{K}_{\mathrm{w}}, \mathrm{K}_{\mathrm{v}}\right.$ and $\left.\mathrm{K}_{\mathrm{m}}\right)$.

$$
k=k_{m}+k_{v}+k_{w}
$$

Then:

$$
\dot{\theta}_{l w}-\dot{\theta}_{b}=\dot{\alpha}, \dot{\theta}_{r w}-\dot{\theta}_{b}=\dot{\beta} \text {. }
$$

After that, it turned as below: 


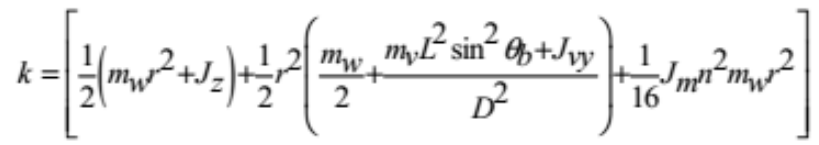

$$
\begin{aligned}
& \left(\dot{\alpha}^{2}+\dot{\beta}^{2}\right)+\left[m_{v} r^{2}+J_{z}+\frac{1}{2}\left(m_{v} L^{2}+J_{v w c}\right)+m_{v} L r \cos \theta_{b}+\frac{1}{2} m_{v} r^{2}\right] \dot{\theta}_{b}^{2} \\
& +\left[m_{w} v^{2}+J_{z}+\frac{1}{2} m_{v} L r \cos \theta_{b}+\frac{1}{2} m_{w^{\prime}}{ }^{2}\right] \dot{\theta}_{b}(\dot{\alpha}+\dot{\beta}) \\
& +\left[\frac{1}{4} m_{w} r^{2}-r^{2}\left(\frac{m_{w}}{2}+\frac{m_{v} L^{2} \sin ^{2} \theta_{b}+J_{v y}}{D^{2}}\right)\right] \dot{\alpha} \dot{\beta}
\end{aligned}
$$

\section{Potential Energy and Energy Wasting}

The total potential energy of system is as below:

$$
U=m_{v} g L \cos \theta_{b}
$$

The total wasting energy is then as below:

$$
\begin{aligned}
G= & \frac{1}{2} \mu_{2}\left[\dot{\alpha}^{2}+\dot{\beta}^{2}+2(\dot{\alpha}+\dot{\beta}) \dot{\theta}_{b}+2 \dot{\theta}_{b}\right]+ \\
& \frac{1}{2} \mu_{2} \dot{\alpha}^{2}+\frac{1}{2} \mu_{2} \dot{\beta}^{2}
\end{aligned}
$$

\section{BELBIC introducing}

Carolucas have suggested BELBIC; it uses the provided network model by the Moren and Balkenius At this time the fuzzy logic, neural networks and genetic algorithm are the advanced tools in controlling applicable programs with complex nonlinear settings (Azimi \& Koofigar, 2013; Guan et al., 2015). It is a nonlinaer controller, a neomorphic controller based on computing learning model.

\section{Literature Review}

Wei An and Li (2013) assessed the Segway robot balance via the PID and LQR controllers and showed that LQR has the better results. Mahler and Haase (2013) explained the robot balance via the Lagrangian Mechanic and designed a system based on it and finally assessed the robot balance based on it. Ruan and Li (2014) designed a controller system based on Ultrasonic sensors. In this method a DSP controller has been used for the robot balance evaluation and a fuzzy method improved the robot balanc. Jamil et al (2014) assessed the robot movement upon to Kinematics and revealed that the robot balance needs a suitable controller to response in a suitable moment.

\section{Suggested Method}

The BELBIC has been used for this aim and the robot will move in Sinusoidal path. The relation structure between the robot and BELBIC has been showed in figure 4.

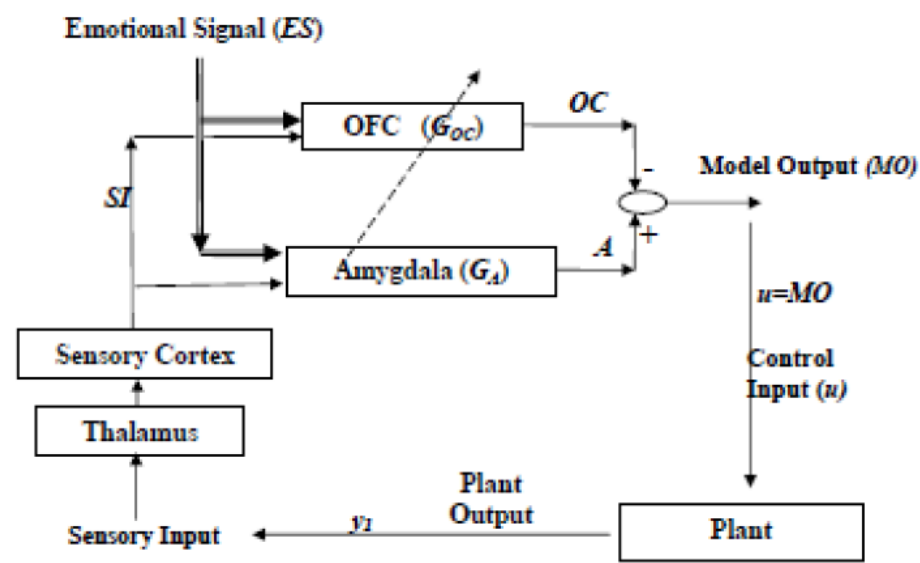

Fig4. The structure of BELBIC relation to Segway robot 
Based on inputs, a network is made called emotion. The data determine the robot movement path, received from the robot sensors and consists of correct path, path deviation and the deviation angle.Seventy percent of data selected as training data, $15 \%$ as test and $15 \%$ as validation data. BELBIC structure has been modeled by neural network with three layer. Robot movement has been modeled for 60 second and figure 5 shows the robot movement path.

\section{Results Evaluation}

The movement path of Segway robot is shown in figure 5. Figure 6 is the present error amount diagram, is shown in 60-second path.
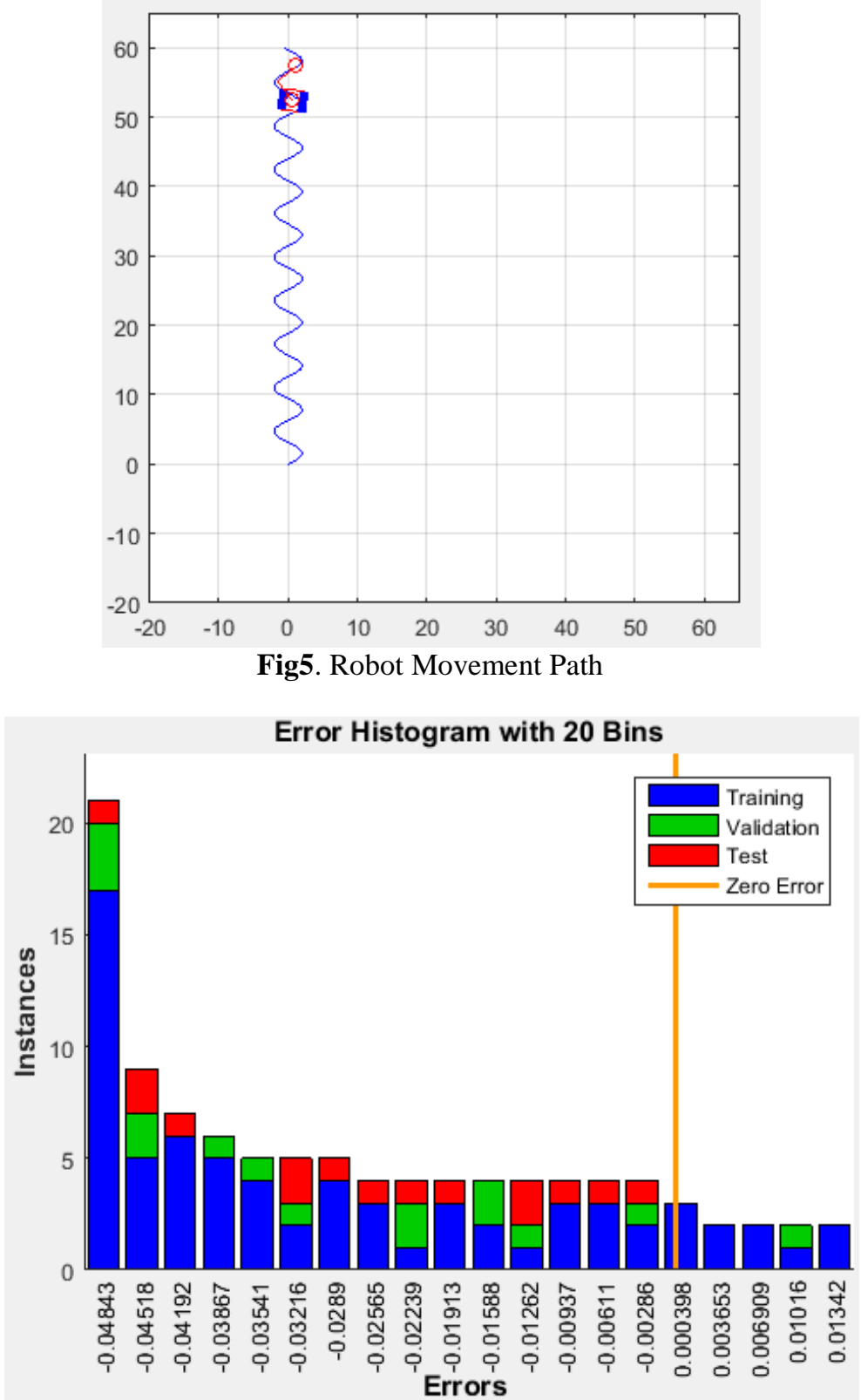

Fig6. Error Amount

In figure 5, the vertical and horizontal coordinates show the time and movement path respectively. Figure six shows during the time the error amount reduces and will decrease until the neural network learns the robot control and minimize the error amount. Figure 7 shows the error path in train and test data. It is obvious that train process done correctly that robot could move correctly in path. 

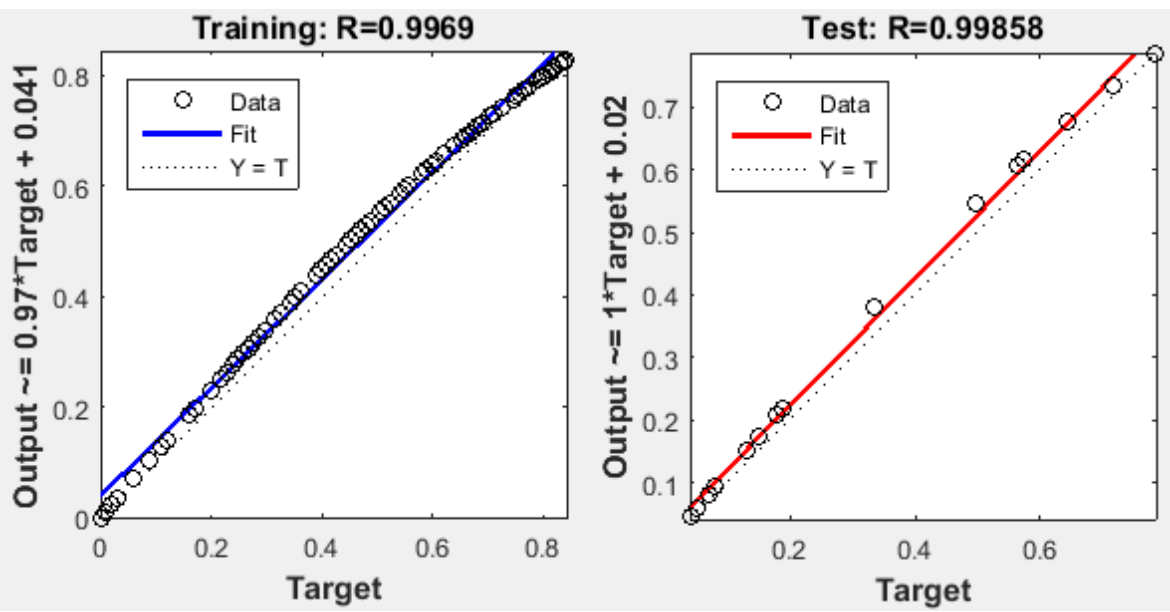

Fig7. Train process for input data to robot controller

Validating the robot movement and train evaluation during the robot movement path, some random points are considered and robot should cross them during the movement path. In this figure, the validation data and movement path have been shown by circle and green line respectively.

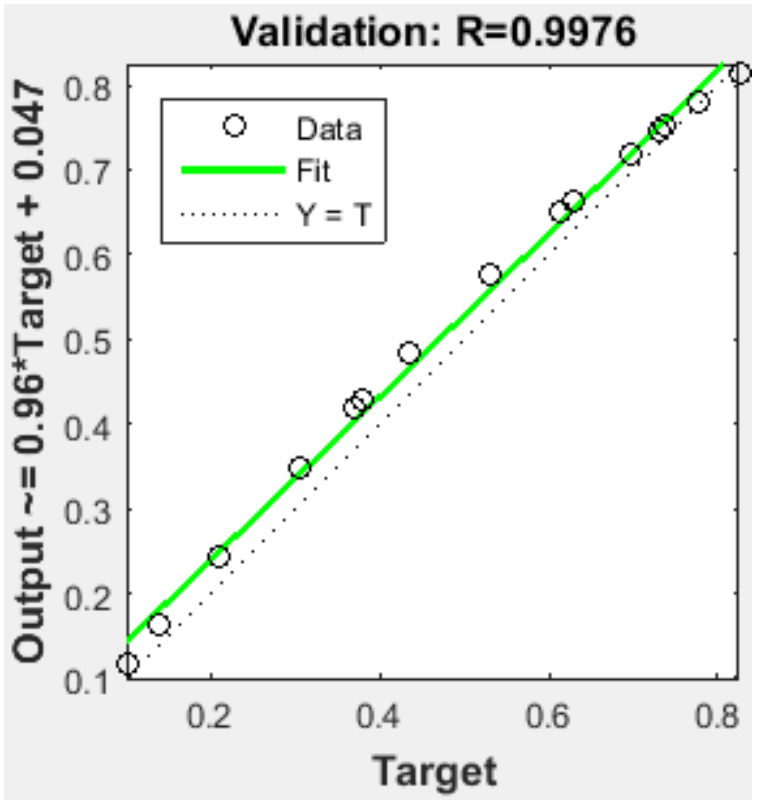

Fig8. Validation Data

\section{Conclusion}

In this article the mechanism of Segway robot has been assessed and its control done by BELBIC controller. Considering the obtained results of simulation, it can be understand that BELBIC controller can regulate the robot movement after the balance keeping and minimize the deviation amount and its error.

\section{References}

[1] An, W., \& Li, Y. (2013, 12-14 Dec. 2013). Simulation and control of a two-wheeled self-balancing robot. Paper presented at the 2013 IEEE International Conference on Robotics and Biomimetics (ROBIO).

[2] Azimi, M. M., \& Koofigar, H. R. (2013, 13-15 Feb. 2013). Model predictive control for a two wheeled self balancing robot. Paper presented at the Robotics and Mechatronics (ICRoM), 2013 First RSI/ISM International Conference on.

[3] Ebrahimi, S., \& Mardany, A. (2015, 7-9 Oct. 2015). Dynamic modeling and construction of a two-wheeled mobile manipulator, part I: Self-balancing. Paper presented at the Robotics and Mechatronics (ICROM), 2015 3rd RSI International Conference on.

[4] Gong, Y., Wu, X., \& Ma, H. (2015, 23-25 Oct. 2015). Research on Control Strategy of Two-Wheeled Self-Balancing Robot. Paper presented at the Computer Science and Mechanical Automation (CSMA), 2015 International Conference on.

[5] Guan, W., Liang, P., \& Gao, H. (2015, 23-25 May 2015). Fault-tolerant control of the self-balancing two-wheeled trolley with actuator saturation. Paper presented at the The 27th Chinese Control and Decision Conference (2015 CCDC).

[6] Jamil, O., Jamil, M., Ayaz, Y., \& Ahmad, K. (2014, 22-24 April 2014). Modeling, control of a two-wheeled self-balancing robot. Paper presented at the Robotics and Emerging Allied Technologies in Engineering (iCREATE), 2014 International Conference on. 
[7] Kim, J., Yoo, J., Bae, J., Kim, C., \& Choi, J. (2015, 20-22 Sept. 2015). Two-Wheeled Self-Balancing Robot Based On LQ-Servo with Reduced Order Observer. Paper presented at the 2015 Fourth International Conference on Information Science and Industrial Applications (ISI)

[8] Mahler, B., \& Haase, J. (2013, 10-13 Nov. 2013). Mathematical model and control strategy of a two-wheeled self-balancing robot. Paper presented at the Industrial Electronics Society, IECON 2013 - 39th Annual Conference of the IEEE.

[9] Ruan, X., \& Li, W. (2014, 3-6 Aug. 2014). Ultrasonic sensor based two-wheeled self-balancing robot obstacle avoidance control system. Paper presented at the 2014 IEEE International Conference on Mechatronics and Automation.

[10] Sadeghian, R., \& Masoule, M. T. (2016, 27-28 Jan. 2016). An experimental study on the PID and Fuzzy-PID controllers on a designed two-wheeled self-balancing autonomous robot. Paper presented at the 2016 th International Conference on Control, Instrumentation, and Automation (ICCIA)

[11] Wang, S., Lu, H., \& Hou, F. (2015, 14-16 Oct. 2015). An improved computing model for a two-wheeled self-balancing vehicle's state determination. Paper presented at the 2015 8th International Congress on Image and Signal Processing (CISP).

[12] Wardoyo, A. S., Hendi, S., Sebayang, D., Hidayat, I., \& Adriansyah, A. (2015, 20-22 May 2015). An investigation on the application of fuzzy and PID algorithm in the two wheeled robot with self balancing system using microcontroller. Paper presented at the Control, Automation and Robotics (ICCAR), 2015 International Conference on 